Fritz K. Winkler

\title{
Amt/MEP/Rh proteins conduct ammonia
}

Received: 18 May 2005 / Accepted: 13 June 2005/Published online: 5 November 2005

(C) Springer-Verlag 2005

\begin{abstract}
The structure determination of the ammonium transport protein AmtB from Escherichia coli strongly indicates that the members of the ubiquitous ammonium transporter/methylamine permease/Rhesus (Amt/ $\mathrm{MEP} / \mathrm{Rh}$ ) protein family are ammonia-conducting channels rather than ammonium ion transporters. The most conserved part of these proteins, apart from the common overall structure with 11 transmembrane helices, is the pore lined by hydrophobic side chains except for two highly conserved histidine residues. A highaffinity ion-binding site specific for ammonium is present at the extracellular pore entry of the Amt/MEP proteins. It is proposed to play an important role in enhancing net transport at very low external ammonium concentrations and to provide discrimination against water. The site is not conserved in the animal Rhesus proteins which are implicated in ammonium homeostasis and saturate at millimolar ammonium concentrations. Many aspects of the biological function of these ammonia channels are still poorly understood and further studies in cellular systems are needed. Likewise, studies with purified, reconstituted Amt/MEP/Rh proteins will be needed to resolve open mechanistic questions and gain a more quantitative understanding of the conduction mechanism in general and for different subfamily representatives.
\end{abstract}

Keywords Ammonium transport - Ammonia channel · AmtB structure $\cdot$ Amt/MEP/Rh proteins $\cdot \mathrm{NH} 3$

F. K. Winkler

Structural Biology, Biomolecular Research,

Paul Scherrer Institut, 5232 Villigen, Switzerland

E-mail: fritz.winkler@psi.ch

Tel.: +41-56-3104258

Fax: + 41-56-3105288

\section{Introduction}

Ammonium $^{1}$ is a key nitrogen source for bacteria, fungi, and plants and becomes assimilated predominantly by glutamine synthetase and glutamate dehydrogenase [42]. The ammonium transporter (Amt) and methylamine permease (MEP) proteins have been shown to be important for nitrogen nutrition at low external ammonium concentrations. In animals, ammonium transport across membranes is important for maintaining ammonium homeostasis [45] and is specifically implicated in the maintenance of $\mathrm{pH}$ balance in the kidney [32, 43, 44]. The selective expression of the human Rhesus $(\mathrm{Rh})$ proteins in cells and tissues involved in such functions supports the notion that they are critically involved in these processes. Sequence comparisons show that the Amt, MEP and Rh proteins represent one large and ubiquitous membrane protein family [24, 29].

The recent structure determination of a first representative of this ubiquitous, so-called ammonium transport family, AmtB from Escherichia coli, carried out independently in two laboratories [19,49] has made it possible to examine the mechanism of ammonium transport at the level of atomic interactions. The main emphasis of this review will be on discussing the new structural insights into the conduction mechanism in relation to the controversial results that have previously been reported on the nature of the transported substrate, uncharged $\mathrm{NH}_{3}$ or cationic $\mathrm{NH}_{4}^{+}$. The structure of AmtB also permits homology modeling within this large family and thus more informative sequence comparisons than hitherto possible.

Key questions with respect to the mechanism of Amt/MEP/Rh-mediated transmembrane ammonium transport concern substrate identity and specificity, the nature and role of substrate-binding sites, the driving

\footnotetext{
${ }^{1}$ Here, the term ammonium is used when no discrimination between $\mathrm{NH} 3$ and $\mathrm{NH}_{4}^{+}$is made. When specificity is required, chemical symbols or the terms ammonia and ammonium ion are used.
} 
force for net transport, the maximum transport rate of a single channel, the possible dependence of this rate on $\mathrm{pH}$ or other variables, and the identification of ratelimiting steps. The new structural information greatly helps to formulate these intrinsic mechanistic questions more precisely and design potentially interesting mutant proteins to answer them. Very importantly, it will also be necessary to develop more reliable, quantitative assays in vitro and in vivo which are and have been prone to misinterpretations because of complicating factors such as passive diffusion or secondary responses that can be induced in living cells.

\section{Structure of AmtB and mechanistic implications}

The trimeric state of purified, detergent-solubilized AmtB [4] and its high stability have provided favorable conditions for the crystallization and high-resolution structure determination of this membrane protein. The structure as determined in different crystal forms by two groups [19, 49] is largely identical except for interesting differences in the local structure of two cytoplasmic loops. One monomer (Fig. 1a) of the tightly packed trimer comprises 11 transmembrane helices which show considerable variation in length, tilt, and internal bends. Their topological arrangement represents a new fold and consists of two pentahelical bundles in opposite arrangement (antiparallel with respect to the membrane normal) and a C-terminal long helix tilted by about $45^{\circ}$. The two pentahelical substructures, probably the result of gene duplication, are related by an approximate local twofold axis, but this relationship is very poor at the sequence level.

A prime interest of the structure determinations was the identification of the substrate translocation pathway
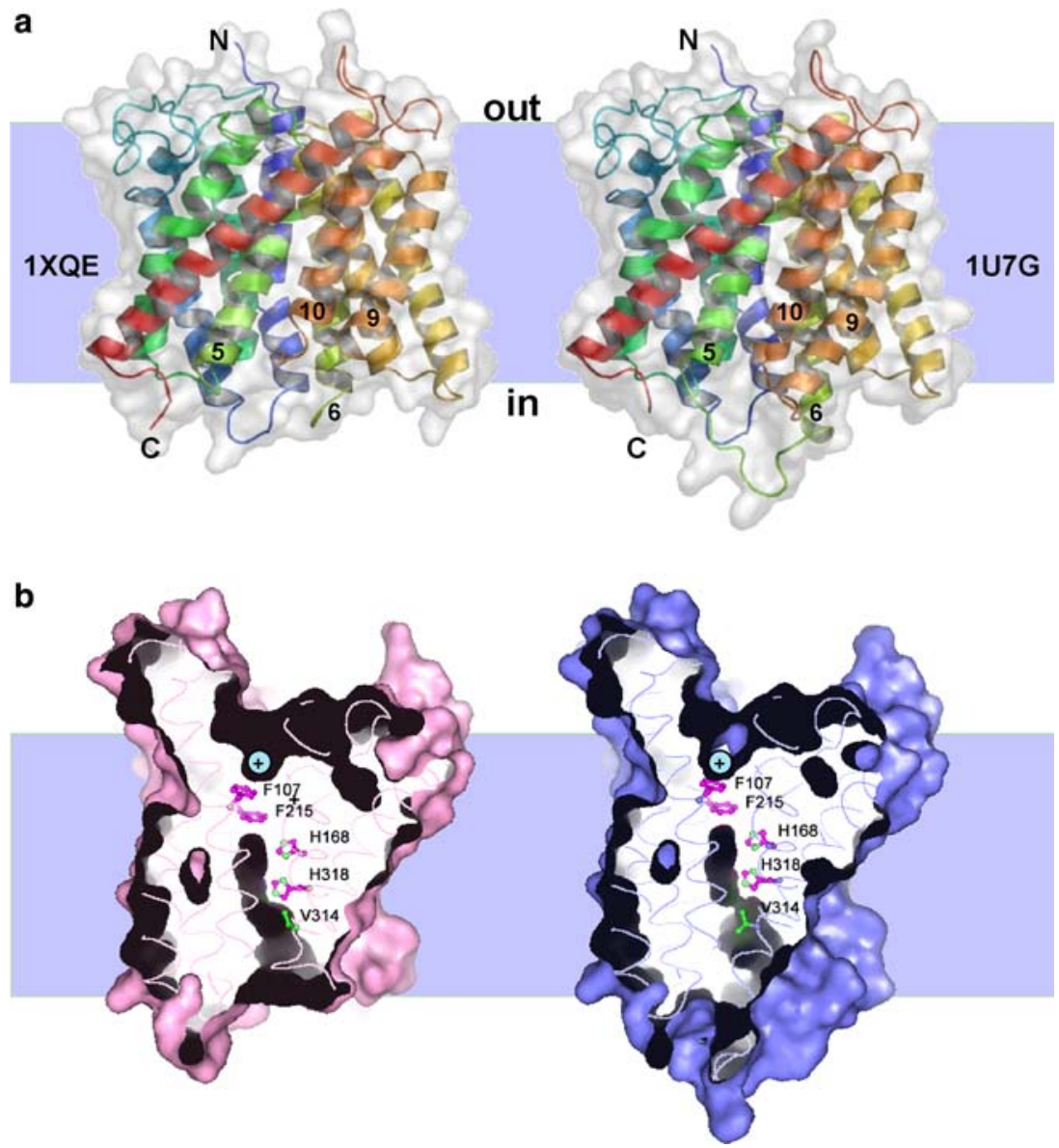

Fig. 1 Monomer structure of AmtB and location of the conduction pore. a Ribbon representation of one AmtB monomer as situated in the membrane with color changing gradually from blue (N-terminus) to red (C-terminus). The corresponding van der Waals surface is shown in light gray. The structures of $1 \mathrm{XQE}$ [49] and 1 U7G [19] are shown side by side to illustrate structural differences on the cytoplasmic side. Symbols $N$ and $C$ mark the Nand C-terminus, respectively, and numbers identify selected transmembrane (TM) helices. The latter are placed at identical positions in the two structures to illustrate the presence of additional helical turns in TM6, TM9, and TM10 in 1U7G. The loops connecting TM5 with TM6 and TM9 with TM10 are disordered in 1XQE. b Cut through the AmtB monomer surface to illustrate the central location and shape of the conduction pores. The extracellular ammonium-binding site (indicated by a plus sign in a blue circle), the constriction at the highly conserved F107 and F215 residues and the special arrangement of the two highly conserved histidine side chains (H168 and H318) lining the center of the pore are indicated. Note the different position of Val314 (shown in green) at the N-terminus of TM10 in the 1XQE and $1 \mathrm{U} 7 \mathrm{G}$ structure, respectively. As a consequence, the pore shows a second constriction below His318 in 1U7G (see text) 
because its structural and chemical characteristics were expected to provide an unambiguous answer as to the chemical nature, $\mathrm{NH}_{3}$ or $\mathrm{NH}_{4}^{+}$, of the permeating species. The pathway, identified by both groups, is through an about $20 \AA$ long narrow pore, which is located in between the two pentahelical domains (Fig. 1b). An ammonium ion-binding site has been identified at the bottom of the shallow depression on the extracellular surface of each monomer. Although the chemical nature of the bound species cannot be safely assigned from the observed electron density, several points strongly support this interpretation. Most convincing is the observation of an elongated density peak (in place of a round one) at this position when ammonium is replaced by methylammonium in the crystal buffer [19]. The presence of an ammonium ion-binding site is consistent with many functional studies showing apparent saturation at low micromolar concentrations for high-affinity Amts [9, $11,21,28,30]$. How can we be sure that this is really a binding site for the ammonium ion and not for $\mathrm{NH}_{3}$ ? First, the binding site has two polar groups, Ser219 $\mathrm{O} \gamma$ and an adjacent water, within hydrogen bonding distance and two aromatic side chains (Phe107, Trp148) at distances favorable for cation- $\pi$ interactions $[19,49]$. Second, if the site were selective for $\mathrm{NH}_{3}$ the affinity would have to be more than 100 times stronger to explain the functional data. This yields an unreasonably high-binding affinity for such a small molecule as already pointed out in the pioneering work of Hackette et al. [12]. The postulated ammonium ion-binding site must, however, be rather selective as no significant inhibition by $\mathrm{Na}^{+}$or $\mathrm{K}^{+}$ions has been observed even at rather high concentrations. Indeed, the combination of three $\mathrm{H}$-bonds and one cation- $\pi$ interaction in a synthetic small molecule ammonium receptor has yielded micromolar affinity for $\mathrm{NH}_{4}^{+}$and a selectivity of nearly 1,000 against $\mathrm{K}^{+}$[6]. The combination of these two types of interactions has also been noted in the biomolecular recognition of substituted ammonium ligands [35].

At its periplasmic entry, the pore is highly constricted by the partly stacked side chains of Phe107 and Phe215 that are strictly conserved in the whole Amt/MEP/Rh family. It is thought that transient structural fluctuations are sufficient to permit frequent passage of small molecules like $\mathrm{NH}_{3}$ or $\mathrm{H}_{2} \mathrm{O}$. It is not known whether these fluctuations limit the maximum rate of conduction. The residues lining the pore are predominantly nonpolar, which is the main structure-based argument to conclude that $\mathrm{NH}_{3}$ and not $\mathrm{NH}_{4}^{+}$is conducted by AmtB. The only exceptions are two centrally located, highly conserved histidines, His168 and His318. They are related by the twofold quasi-symmetry, and their adjacent imidazole rings (Fig. 1b) are arranged such that a hydrogen bond is formed between their two $\delta 1$ nitrogens. The remarkable conservation of this imidazole pair arrangement has given rise to speculations that it may have a role in the deprotonation of ammonium ions before they cross the central part of the channel as uncharged $\mathrm{NH}_{3}$ molecules [49]. However, this would require that ammonium ions can easily penetrate into the pores up to these positions. Interestingly, Saccharomyces cerevisiae Mep1p and Mep3p, and a few other members of the Amt/MEP subfamilies have a glutamate residue replacing the conserved His168 in transmembrane helix 5 (TM5). In its neutral form, a glutamate could indeed occupy about the same space and make the same hydrogen bonding interactions.

Important differences between the AmtB structures observed in the different crystal forms occur only on the cytoplasmic side (Fig. 1a). Most of the residues in the long loop connecting TM5/TM6 are disordered in the two structures reported by Zheng [49] and the cytoplasmic ends of TM9 and TM10 as well as their connection are also affected by disorder (see legend Fig. 1). Independent of the cause of this difference (different detergent, different $\mathrm{pH}$, and different crystal packing) an intrinsic structural flexibility of these segments is indicated. In the fully ordered state [19], TM10 starts with a $3_{10}$ helical turn which generates a narrow constriction at the cytoplasmic exit around Val314 before the channel opens into the cytoplasmic vestibule (Fig. 1b). In the $1 \mathrm{XQF}$ structure [49] the same $3_{10}$ helical turn conformation is observed, but the nine preceding residues are disordered. In the $1 \mathrm{XQE}$ structure, from yet another crystal form [49] as shown in Fig. 1, residues 309-314 at the N-terminus of TM10 assume a different, nonhelical conformation, which widens the cytoplasmic constriction by about $2 \AA$. Furthermore, it renders it more polar because the main chain carbonyl group of Cys312 points into the pore in place of the Val314 side chain. It has been speculated [49] that this more open-structural state may correspond to the conducting state of the channel and that the fully ordered but more closed state might be induced by the binding of the regulatory protein GlnK known to inactivate AmtB conduction [8, 17, 19].

The C-terminal 20 residues are disordered in all crystal forms suggesting that they are not essential for conduction. In agreement, truncations after TM11 have no or little effect on ammonium conduction but appear to interfere with different regulatory mechanisms $[3,8$, 37]. In addition, a specific $G$ to $D$ mutation (G393 in AmtB) in Meplp of Saccharomyces [26], MeaA of Aspergillus nidulans [31] and LeAmt1:1 from tomato [25] leads to inhibition of ammonium transport. Because of its trans-dominant effect it has been suggested that this mutation may lead to inhibitory interactions in oligomers but the structural mechanism remains rather unclear at this point.

\section{Ammonia diffusion through lipid bilayers and cellular membranes}

The so-called passive diffusion of small neutral molecules across lipid bilayers is generally characterized by the diffusional membrane permeability $P_{\mathrm{d}}$ which permits to express net flux (per area) simply as the product $P_{\mathrm{d}} \cdot \Delta c$ with $\Delta \mathrm{c}$ being the concentration gradient of the 
permeating molecule. It is well known that $P_{\mathrm{d}}$ for molecules such as water or ammonia can vary considerably with lipid composition and cell type [7, 14, 20, 22]. For equilibration experiments it can be important to have some estimate of the time needed for equilibration of a concentration gradient across the membrane. The time course of diffusional equilibration of a permeable solute across the membrane can be described by a single exponential with time constant $1 / \tau=V /\left(A \cdot P_{\mathrm{d}}\right)$ where $V$ and $A$ are volume and area of the permeated container $[36,41]$. For small spherical containers where internal diffusion times can be neglected, the time needed for equilibration, $t(95 \%)$ then becomes about $r / P_{\mathrm{d}}$. With a typical $\mathrm{NH}_{3}$ permeability coefficient of $10^{-3} \mathrm{~cm} / \mathrm{s}$, equilibration across the membrane of a liposome $(100 \mathrm{~nm}$ diameter) is predicted to take only milliseconds and still less than $1 \mathrm{~s}$ for a $10 \mu \mathrm{m}$ diameter cell. Stopped-flow techniques are needed to follow such rapid equilibration, but it is well known that the observed kinetics can be limited by unstirred layer effects [7]. Equilibration times in the minute range are expected for the much larger frog oocytes (typically $0.5-1 \mathrm{~mm}$ diameter) but in this case intracellular diffusional mixing may no longer be negligible [36].

$\mathrm{NH}_{3}$ influx is alkalizing and will increase the $\mathrm{pH}$ of the container by an amount depending on its buffering capacity. Monitoring this $\mathrm{pH}$ change via a $\mathrm{pH}$ sensitive fluorescent dye has been used to follow $\mathrm{NH}_{3}$ equilibration processes in liposomes [22] or resealed red blood cell ghosts [34]. In oocytes, rapid alkalinization can apparently induce secondary responses like the activation of other transport systems, pumps, or channels [5].

\section{Protein mediated ammonia conduction}

Based on the predominantly nonpolar nature of the pore of AmtB, both structural studies have concluded that conduction of the charged form appears energetically highly unfavorable and that $\mathrm{NH}_{3}$ must be the permeating species as suggested by the Kustu group for some time [37, 39]. Stopped-flow experiments with purified AmtB reconstituted into proteoliposomes [19] and a recent in vivo functional characterization of E. coli AmtB are also consistent with this view [18]. Sequence alignments with representatives from the MEP and Rh families [19, 49] show that the nonpolar nature of the pore lining residues is highly conserved, which strongly suggests that all

$$
\mathrm{P}+\left(\mathrm{NH}_{4}^{+}\right)_{\text {out }} \underset{\mathrm{k}_{-1}}{\stackrel{\mathrm{k}_{1}}{\longrightarrow}} \mathrm{P}-\left(\mathrm{NH}_{4}^{+}\right) \stackrel{\mathrm{k}_{2}}{\longrightarrow} \mathrm{P}+\left(\mathrm{NH}_{3}\right)_{\text {in }}+\mathrm{H}_{\text {out }}^{+}
$$

Scheme 1 Kinetic scheme for AmtB mediated ammonia influx (out $\rightarrow$ in) driven by the $\mathrm{NH}_{3}$ chemical gradient. The back reaction $\left(k_{-2}\right)$ is ignored as the intracellular ammonia concentration is assumed to remain much below the equilibrium concentration by metabolic trapping. At saturation, the rate is given by $k_{2}$ which members of the Amt/MEP/Rh family conduct uncharged $\mathrm{NH}_{3}$.

While Amt/MEP/Rh ammonia channels appear generally needed to increase transmembrane $\mathrm{NH}_{3}$ flux, their relative contribution to total flux will (a) depend on their membrane density and intrinsic conduction rate and (b) on the permeability of the bilayer, on total ammonium concentration and on $\mathrm{pH}$. There are two situations where a need for protein-mediated $\mathrm{NH}_{3}$ conduction appears biologically indicated. The first, relevant for nutrition, is when the external ammonium concentration becomes so low that passive diffusion becomes limiting for growth. The second, relevant for animal cells, is when lipid-mediated $\mathrm{NH}_{3}$ diffusion does not provide sufficient transport capacity to satisfy physiological needs or when ammonium transport needs to be tightly regulated.

\section{Amt/MEP-mediated ammonia uptake for nutrition}

Here, I want to examine whether ammonia-conducting Amt/MEP channels can fulfill the nutritional requirements under nitrogen starvation. In contrast to the prevailing view of interpreting cellular methylammonium accumulation as Amt/MEP-mediated secondary transport as reviewed by von Wirén and Merrick [42], Kustu and collaborators [37, 39, 40] have attributed this accumulation to metabolic trapping by glutamine synthetase or to trapping in acidic vacuoles in the case of fungi. They proposed instead that Amt/MEP proteins simply mediate bi-directional $\mathrm{NH}_{3}$ transport and this view is now strongly supported by the structural results. The activation of ammonia channels under ammonium starvation can perhaps be considered as the least disturbing way for the cell to compensate for insufficient passive ammonia influx. In contrast, switching to $\mathrm{NH}_{4}^{+}$ influx upon starvation would affect the membrane potential and require the activation of mechanisms compensating for its depolarization.

For an E. coli strain carrying an $a m t B$ disruption, the limiting external $\mathrm{NH}_{3}$ concentration for normal growth was found to be below $100 \mathrm{nM}$ [38]. The AmtB-mediated transport capacity equivalent to passive influx at this concentration has been estimated to require conduction rates of $10^{1}-10^{4}$ molecules per channel per second [49]. Using a simple model [15] to estimate the diffusion limited rate for entering a pore $\left(\phi_{\text {pore mouth }}\right)$ of $4 \AA$ diameter yields a maximum of about 60 molecules per

may depend on the composition of the external solution. Below saturation the forward rate per channel can be approximated by $\mathrm{d}\left(\mathrm{NH}_{3}\right)_{\text {out }} / \mathrm{d} t=\left(k_{1} k_{2} /\left(k_{-1}+k_{2}\right)\right) \cdot\left[\mathrm{NH}_{4}^{+}\right]_{\text {out }}$ and with $k_{2}>k_{-1}$ it becomes approximately $k_{1} \cdot\left[\mathrm{NH}_{4}{ }^{+}\right]$ 
second at a concentration of $100 \mathrm{nM}$. This appears short of what is needed, as not every molecule reaching the pore entry will make it through the channel.

I propose that the extracellular ammonium-binding site is essential in overcoming this limitation. The familiar Michaelis-Menten formalism can be used to describe binding to the external $\mathrm{NH}_{4}^{+}$site followed by a reaction comprising deprotonation and diffusion through the pore (Scheme 1).

As shown in Scheme 1 the forward rate becomes dependent on the ammonium ion concentration below saturation $\left(\left[\mathrm{NH}_{4}^{+}\right]<K_{\mathrm{m}}=\left(k_{-1}+k_{2}\right) / k_{1}\right)$ and it becomes diffusion limited with $k_{2}>k_{-1}$. Under these conditions the relative rate of the two processes (with and without $\mathrm{NH}_{4}^{+}$binding site) is approximately given by $\left(\left[\mathrm{NH}_{4}^{+}\right]_{\text {out }} /\left(\left[\mathrm{NH}_{3}\right]_{\text {out }}\right.\right.$, which is over 100 at neutral $\mathrm{pH}$ and even higher at acidic $\mathrm{pH}$. Apparent $K_{\mathrm{i}}$ or $K_{\mathrm{m}}$ $\left(\mathrm{NH}_{4}^{+}\right)$values of Amt/MEP ammonium transport systems are typically in the range of $1-50 \mu \mathrm{M}$. With $k_{2}$ values of $10^{3}-10^{4} \mathrm{~s}^{-1}$, the forward reaction would thus be diffusion limited below saturation. An important question concerns the nature of the process limiting the value of $k_{2}$. Direct proton transfer to water is estimated to be rather slow [10] and ammonium ion deprotonation probably requires base catalysis. As no suitable functional group is near the ammonium ion-binding site in AmtB, small basic molecules with suitable $\mathrm{p} K_{\mathrm{a}}$ values (possibly phosphates, carbonates, etc.) would have to be present at sufficient concentration in the periplasmic space. Dissociation into the pore rather than deprotonation itself would appear to be rate-limiting in such a scenario. Alternatively, the two conserved pore histidines are attractive candidates to serve as transient proton acceptors. However, this would require that the proton could rapidly diffuse back to the bulk solution. It is not obvious how this should happen and I do not consider such a mechanism very likely despite the intriguing arrangement of the two conserved pore histidines.

To confirm the proposed role of the $\mathrm{NH}_{4}^{+}$binding site, the kinetics of Amt/MEP-mediated ammonia conduction will need to be investigated with purified proteins reconstituted into liposomes or planar bilayers. It should be particularly interesting to determine the maximum forward conduction rate $\left(k_{2}\right)$, its dependence on $\mathrm{pH}$ and the possible importance of buffer components for enhancing the rate by general base catalysis.

\section{Rh-mediated ammonia transport in animal cells}

The role of the animal $\mathrm{Rh}$ proteins is thought to be in both ammonium uptake and excretion and particularly in transepithelial transport depending on the specific physiological needs in different tissues and organs. Here, I discuss whether their proposed function as ammonia channels is consistent with functional data and in what respect they differ from the Amt/MEP proteins.
The human $\mathrm{Rh}$ glycoprotein family consists of the highly polymorphic $R H D$ and $R H C E$ genes, the carriers of the $\mathrm{Rh}$ blood group antigens, as well as of the erythroid $R H A G$ and the nonerythroid $R H B G$ and $R H C G$ genes [46]. The RhD, RhCE, and RhAG proteins form a heteromeric, most likely heterotrimeric, complex but only RhAG has been shown to function in ammonium transport [27, 48]. RhD and RhCE differ in some otherwise highly conserved pore residues. In particular, the highly conserved Asp/His pairs of segments Asp160 His168 and Asp310-His18, which shape functionally critical regions of the pore on both sides are replaced by Tyr/Tyr and His/Phe, respectively, and it is not surprising that RhD and RhCE appear to be nonfunctional. The two highly homologous nonerythroid glycoproteins, RhBG and RhCG, are selectively expressed in a number of tissues such as skin, kidney, liver, testis, central nervous system, and gastrointestinal tract $[13,16,33]$. Where implicated in transepithelial transport, they show polarized expression with RhBG localized in the basolateral and RhCG in the apical membrane segment, respectively.

Functional studies with RhAG, RhBG, and RhCG have been carried out by expression in oocytes [2, 23, 32, 47], and in ammonium transport deficient S. cerevisiae $[27,48]$. They have been interpreted in terms of $\mathrm{NH}_{4}^{+}$ transport [27, 32], influx of both $\mathrm{NH}_{3}$ and $\mathrm{NH}_{4}^{+}$([2] and $\mathrm{NH}_{4}^{+} / \mathrm{H}^{+}$exchange $[23,47,48]$. Electroneutral $\mathrm{NH}_{4}^{+} /$ $\mathrm{H}^{+}$exchange has been inferred mainly from the $\mathrm{pH}$ dependence of substrate uptake rates but, as stated by the authors, the data do not really distinguish between $\mathrm{NH}_{3}$ influx and $\mathrm{NH}_{4}^{+} / \mathrm{H}^{+}$exchange. The induction of current observed in some studies with frog oocytes most likely is due to the presence of endogenous $\mathrm{NH}_{4}^{+}$permeable channels that are activated by local alkalinization upon $\mathrm{NH}_{3}$ influx [5]. In the absence of heterologously expressed ammonia channels, this response to ammonia influx is seen only above about $1 \mathrm{mM}$ external $\mathrm{NH}_{4}^{+}$at $\mathrm{pH} 7.5[5,23]$. The significant currents seen in RhBG [32] and RhCG oocytes [2] at even lower concentrations may, therefore, be the consequence of $\mathrm{RhBG} / \mathrm{RhCG}$-enhanced $\mathrm{NH}_{3}$ permeability rather than proof of $\mathrm{NH}_{4}^{+}$transport. All the studies with heterologously expressed $\mathrm{Rh}$ proteins appear compatible with their functioning as $\mathrm{NH}_{3}$ channels.

RhAG, RhBG, and RhCG protein mediated methylammonium uptake in oocytes shows saturation with apparent $K_{\mathrm{m}}$ values in the low millimolar range and is effectively inhibited by ammonium at similar concentrations [23, 47]. Trp148 and Ser219, both of which are important for $\mathrm{NH}_{4}^{+}$binding in the Amt/ MEP proteins are both replaced by aliphatic residues $(\mathrm{V}, \mathrm{I}$ or $\mathrm{L})$ in the $\mathrm{Rh}$ proteins. Consistent with an apparently much reduced affinity, the structure of the AmtB ammonium ion-binding site is not conserved in the $\mathrm{Rh}$ proteins. A lower ammonium ion affinity of the $\mathrm{Rh}$ proteins is functionally plausible as they mediate ammonia conduction typically at millimolar rather than micromolar ammonium concentrations. Analysis 
of RhAG-mediated ammonia influx into red blood cells indicates a conduction rate of about $3 \times 10^{4} \mathrm{~s}^{-1}$ at saturation [34].

\section{Conclusions and outlook}

The pore structure of AmtB strongly indicates that the members of the Amt/MEP/Rh family conduct the uncharged ammonia molecule. Therefore, the driving force for net ammonium transport by these proteins is a chemical $\mathrm{NH}_{3}$ gradient and not the electrochemical ammonium ion gradient as has been the prevailing view not long ago. Conduction occurs through a narrow and locally highly constricted pore. It does not appear to involve a major structural change coupled to single molecule translocation as is usually the case for membrane transporters. The designation "ammonium transporters" that has commonly been used for the Amt/ $\mathrm{MEP} / \mathrm{Rh}$ proteins is, therefore, rather misleading and following Khademi et al. [19] should be replaced by the term ammonia channels. It appears that nature makes use of these membrane channels in situations where lipid-mediated, low capacity $\mathrm{NH}_{3}$ diffusion is not sufficiently effective or needs to be regulated. This is reminiscent of the function of aquaporins and it is an amusing coincidence that Peter Agre and his collaborators stumbled upon aquaporin 1 when studying the $\mathrm{Rh}$ blood group antigens [1]. In contrast to aquaporins, the concentration of the physiological substrate $\left(\mathrm{NH}_{3}\right)$ is orders of magnitude smaller in the case of the Amt/Mep/ $\mathrm{Rh}$ channels. Therefore, in order to match diffusionlimited rates for arriving at the pore, their conduction rate need not be as high as that of aquaporins. The extracellular high-affinity ammonium ion-binding sites of the Amt/MEP proteins appears needed for scavenging ammonium at very low concentrations and permit enhanced net $\mathrm{NH}_{3}$ transport under these conditions. It may also provide discrimination against water which otherwise might compete too effectively with ammonia. The $\mathrm{Rh}$ proteins also provide enhanced ammonia transport capacity but function at considerably higher ammonium concentrations. It remains to be seen whether their lower affinity ammonium ion-binding site correlates with an enhanced channel conduction rate.

Many questions regarding the biological function and molecular mechanism of Amt $/ \mathrm{MEP} / \mathrm{Rh}$ proteins remain open. In many organisms multiple paralogues are present but their specific roles are generally poorly understood. Some fungal members are also implicated in regulating morphogenesis [3]. Ammonia conduction, particularly in animals, may also be coupled to other transport systems which can result in net ammonium transport as seems to be the case for urinary acid secretion. Little is known how different pathways for ammonium transmembrane transport are coordinated in cells and further investigations in living cells are certainly needed. However, I strongly believe that we also have much to learn from in vitro studies with purified
$\mathrm{Amt} / \mathrm{MEP} / \mathrm{Rh}$ proteins and selected mutants in order to define their intrinsic functional characteristics more precisely. The structure determination of $\mathrm{AmtB}$ has certainly given a boost to such activities.

Acknowledgments I thank Lei Zheng for providing Fig. 1 and Xiao-Dan Li and Lei Zheng for critical comments on the manuscript. The author's research was supported by the Swiss National Science Foundation within the framework of the National Center of Competence in Research (NCCR) in Structural Biology.

\section{References}

1. Agre P (2004) Aquaporin water channels (Nobel Lecture). Angew Chem Int Ed Engl 43:4278-4290

2. Bakouh N, Benjelloun F, Hulin P, Brouillard F, Edelman A, Cherif-Zahar B, Planelles $\mathrm{G}$ (2004) $\mathrm{NH}_{3}$ is involved in the $\mathrm{NH}_{4}^{+}$transport induced by the functional expression of the human Rh C glycoprotein. J Biol Chem 279:15975-15983

3. Biswas K, Morschhauser J (2005) The Mep2p ammonium permease controls nitrogen starvation-induced filamentous growth in Candida albicans. Mol Microbiol 56:649-669

4. Blakey D, Leech A, Thomas GH, Coutts G, Findlay K, Merrick M (2002) Purification of the Escherichia coli ammonium transporter AmtB reveals a trimeric stoichiometry. Biochem $\mathbf{J}$ 364:527-535

5. Boldt M, Burckhardt G, Burckhardt BC (2003) $\mathrm{NH}(4)(+)$ conductance in Xenopus laevis oocytes. III. Effect of $\mathrm{NH}(3)$. Pflugers Arch 446:652-657

6. Chin J, Oh J, Jon SY, Park SH, Walsdorff C, Stranix B, Ghoussoub A, Lee SJ, Chung HJ, Park SM, Kim K (2002) Tuning and dissecting electronic and steric effects in ammonium receptors: nonactin vs artificial receptors. J Am Chem Soc 124:5374-5379

7. Cooper GJ, Zhou Y, Bouyer P, Grichtchenko II, Boron WF (2002) Transport of volatile solutes through AQP1. J Physiol 542:17-29

8. Coutts G, Thomas G, Blakey D, Merrick M (2002) Membrane sequestration of the signal transduction protein GlnK by the ammonium transporter AmtB. EMBO J 21:536-545

9. D’Apuzzo E, Rogato A, Simon-Rosin U, El AH, Barbulova A, Betti M, Dimou M, Katinakis P, Marquez A, Marini AM, Udvardi MK, Chiurazzi M (2004) Characterization of three functional high-affinity ammonium transporters in Lotus japonicus with differential transcriptional regulation and spatial expression. Plant Physiol 134:1763-1774

10. Eigen M (2005) Proton transfer, acid-base catalysis, and enzymatic hydrolysis. Angew Chem Int Ed Engl 3:1-72

11. Gazzarrini S, Lejay L, Gojon A, Ninnemann O, Frommer WB, von WN (1999) Three functional transporters for constitutive, diurnally regulated, and starvation-induced uptake of ammonium into Arabidopsis roots. Plant Cell 11:937-948

12. Hackette SL, Skye GE, Burton C, Segel IH (1970) Characterization of an ammonium transport system in filamentous fungi with methylammonium-14C as the substrate. J Biol Chem 245:4241-4250

13. Handlogten ME, Hong SP, Zhang L, Vander AW, Steinbaum ML, Campbell-Thompson M, Weiner ID (2005) Expression of the ammonia transporter proteins, $\mathrm{Rh} B$ Glycoprotein and $\mathrm{Rh}$ C Glycoprotein, in the intestinal tract. Am J Physiol Gastrointest Liver Physiol 288(5):G1036-G1047

14. Hill WG, Zeidel ML (2000) Reconstituting the barrier properties of a water-tight epithelial membrane by design of leafletspecific liposomes. J Biol Chem 275:30176-30185

15. Hille B (2001) Ion channels of excitable membranes. Sinauer Associates, Sunderland, p 354

16. Huang CH, Liu PZ (2001) New insights into the Rh superfamily of genes and proteins in erythroid cells and nonerythroid tissues. Blood Cells Mol Dis 27:90-101 
17. Javelle A, Severi E, Thornton J, Merrick M (2004) Ammonium sensing in Escherichia coli. Role of the ammonium transporter $\mathrm{AmtB}$ and $\mathrm{AmtB}-\mathrm{G} \operatorname{lnK}$ complex formation. J Biol Chem 279:8530-8538

18. Javelle A, Thomas G, Marini AM, Kramer R, Merrick M (2005) In vivo functional characterisation of the E. coli ammonium channel AmtB: evidence for metabolic coupling of AmtB to glutamine synthetase. Biochem J 390(Pt 1):215-222

19. Khademi S, O'Connell J III, Remis J, Robles-Colmenares Y, Miercke LJ, Stroud RM (2004) Mechanism of ammonia transport by Amt/MEP/Rh: structure of $\mathrm{AmtB}$ at $1.35 \mathrm{~A}$. Science 305:1587-1594

20. Kikeri D, Sun A, Zeidel ML, Hebert SC (1989) Cell membranes impermeable to $\mathrm{NH}_{3}$. Nature 339:478-480

21. Kleiner D (1985) Bacterial ammonium transport. FEMS Microbiol Rev 32:87-100

22. Lande MB, Donovan JM, Zeidel ML (1995) The relationship between membrane fluidity and permeabilities to water, solutes, ammonia, and protons. J Gen Physiol 106:67-84

23. Ludewig U (2004) Electroneutral ammonium transport by basolateral rhesus B glycoprotein. J Physiol 559:751-759

24. Ludewig U, von Wiren N, Rentsch D, Frommer WB (2001) Rhesus factors and ammonium: a function in efflux?. Genome Biol 2:1-5

25. Ludewig U, Wilken S, Wu B, Jost W, Obrdlik P, El BM, Marini AM, Andre B, Hamacher T, Boles E, von WN, Frommer WB (2003) Homo- and hetero-oligomerization of ammonium transporter-1 $\mathrm{NH}_{4}$ uniporters. J Biol Chem 278:45603-45610

26. Marini AM, Andre B (2000) In vivo $N$-glycosylation of the mep2 high-affinity ammonium transporter of Saccharomyces cerevisiae reveals an extracytosolic $\mathrm{N}$-terminus. Mol Microbiol 38:552-564

27. Marini AM, Matassi G, Raynal V, Andre B, Cartron JP, Cherif-Zahar B (2000) The human Rhesus-associated RhAG protein and a kidney homologue promote ammonium transport in yeast. Nat Genet 26:341-344

28. Marini AM, Soussi-Boudekou S, Vissers S, Andre B (1997) A family of ammonium transporters in Saccharomyces cerevisiae. Mol Cell Biol 17:4282-4293

29. Marini AM, Urrestarazu A, Beauwens R, Andre B (1997) The $\mathrm{Rh}$ (rhesus) blood group polypeptides are related to $\mathrm{NH}_{4}^{+}$ transporters. Trends Biochem Sci 22:460-461

30. Meier-Wagner J, Nolden L, Jakoby M, Siewe R, Kramer R, Burkovski A (2001) Multiplicity of ammonium uptake systems in Corynebacterium glutamicum: role of Amt and AmtB. Microbiology 147:135-143

31. Monahan BJ, Unkles SE, Tsing IT, Kinghorn JR, Hynes MJ, Davis MA (2002) Mutation and functional analysis of the Aspergillus nidulans ammonium permease MeaA and evidence for interaction with itself and MepA. Fungal Genet Biol 36:3546

32. Nakhoul NL, Dejong H, Abdulnour-Nakhoul SM, Boulpaep EL, Hering-Smith K, Hamm LL (2005) Characteristics of renal Rhbg as an NH4(+) transporter. Am J Physiol Renal Physiol 288:F170-F181
33. Nakhoul NL, Hamm LL (2004) Non-erythroid Rh glycoproteins: a putative new family of mammalian ammonium transporters. Pflugers Arch 447:807-812

34. Ripoche P, Bertrand O, Gane P, Birkenmeier C, Colin Y, Cartron JP (2004) Human Rhesus-associated glycoprotein mediates facilitated transport of $\mathrm{NH}(3)$ into red blood cells. Proc Natl Acad Sci USA 101:17222-17227

35. Scrutton NS, Raine AR (1996) Cation-pi bonding and aminoaromatic interactions in the biomolecular recognition of substituted ammonium ligands. Biochem J 319(Pt 1):1-8

36. Sehy JV, Banks AA, Ackerman JJ, Neil JJ (2002) Importance of intracellular water apparent diffusion to the measurement of membrane permeability. Biophys J 83:2856-2863

37. Soupene E, Chu T, Corbin RW, Hunt DF, Kustu S (2002) Gas channels for $\mathrm{NH}(3)$ : proteins from hyperthermophiles complement an Escherichia coli mutant. J Bacteriol 184:3396-3400

38. Soupene E, He L, Yan D, Kustu S (1998) Ammonia acquisition in enteric bacteria: physiological role of the ammonium methylammonium transport B (AmtB) protein. Proc Natl Acad Sci USA 95:7030-7034

39. Soupene E, Lee H, Kustu S (2002) Ammonium/methylammonium transport (Amt) proteins facilitate diffusion of $\mathrm{NH}_{3}$ bidirectionally. Proc Natl Acad Sci USA 99:3926-3931

40. Soupene E, Ramirez RM, Kustu S (2001) Evidence that fungal MEP proteins mediate diffusion of the uncharged species $\mathrm{NH}(3)$ across the cytoplasmic membrane. Mol Cell Biol 21:5733-5741

41. Verkman AS (1995) Optical methods to measure membrane transport processes. J Membr Biol 148:99-110

42. von Wirén N, Merrick M (2004) Regulation and function of ammonium carriers in bacteria, fungi, and plants. Trends Curr Genet 9:95-120

43. Wall SM (2003) Mechanisms of $\mathrm{NH}_{4}^{+}$and $\mathrm{NH}_{3}$ transport during hypokalemia. Acta Physiol Scand 179:325-330

44. Weiner ID (2004) The Rh gene family and renal ammonium transport. Curr Opin Nephrol Hypertens 13:533-540

45. Weiner ID, Verlander JW (2003) Renal and hepatic expression of the ammonium transporter proteins, Rh B Glycoprotein and Rh C Glycoprotein. Acta Physiol Scand 179:331-338

46. Westhoff CM (2004) The Rh blood group system in review: a new face for the next decade. Transfusion 44:1663-1673

47. Westhoff CM, Ferreri-Jacobia M, Mak DO, Foskett JK (2002) Identification of the erythrocyte $\mathrm{Rh}$ blood group glycoprotein as a mammalian ammonium transporter. J Biol Chem 277:12499-12502

48. Westhoff CM, Siegel DL, Burd CG, Foskett JK (2004) Mechanism of genetic complementation of ammonium transport in yeast by human erythrocyte Rh-associated glycoprotein. J Biol Chem 279:17443-17448

49. Zheng L, Kostrewa D, Bernèche S, Winkler FK, Li X-D (2004) The crystal structure of AmtB of E. coli suggests a mechanism for ammonia transport. Proc Natl Acad Sci USA 101:1709017095 